\title{
O impacto do biofilme dental e saburra lingual em pacientes internados em uma UTI em Manaus/AM
}

\author{
The impact of dental biofilm and tongue coating on patients admitted to an ICU in Manaus/AM \\ El impacto de la biopelícula dental y el recubrimiento de la lengua en pacientes ingresados en una \\ UCI en Manaus/AM
}

Recebido: 19/06/2021 | Revisado: 25/06/2021 | Aceito: 27/06/2021 | Publicado: 11/07/2021

\author{
Gabriela de Sousa Soares \\ ORCID: https://orcid.org/0000-0002-2609-5592 \\ Universidade Paulista, Brasil \\ E-mail: gabrielasoares12@hotmail.com \\ Hannah Luiza Batista Almeida \\ ORCID: https://orcid.org/0000-0003-3487-8201 \\ Universidade Paulista, Brasil \\ E-mail: hannah_batista@ hotmail.com \\ Augusto Arcemiro Bittencourt \\ ORCID: https://orcid.org/0000-0002-2542-7655 \\ Universidade do Estado do Amazonas, Brasil \\ E-mail: augustobttncrt@gmail.com \\ Nely Cristina Medeiros Caires \\ ORCID: https://orcid.org/0000-0003-3094-4064 \\ Universidade Paulista, Brasil \\ E-mail: nelycaires@gmail.com
}

\begin{abstract}
Resumo
Este trabalho descreve a condição bucal de pacientes internados em unidade de terapia intensiva (UTI) de um hospital público em Manaus-Amazonas com diagnóstico de pneumonia nosocomial. Realizou-se um estudo de natureza transversal, quantitativa, em pacientes hospitalizados com idade superior a 18 anos, impossibilitados de autocuidado e com diagnóstico clínico de pneumonia. A avaliação clínica da cavidade bucal dividiu-se em dois momentos: inicial (até 48 horas da internação) e após 72 horas. Foram avaliados o índice de placa (IP), a condição da mucosa, a presença ou não de prótese dentária, o número de dentes presentes e o índice de saburra lingual. Dos vinte pacientes avaliados, $60 \%$ eram do gênero feminino e a faixa etária predominante entre 70 e 90 anos de idade (35\%). 13 pacientes encontravamse em VMI (Ventilação Mecânica Induzida) e 7 apresentavam-se em ar ambiente. Houve melhora dos índices de Placa (detecção de biofilme dental) e presença de saburra lingual após 72 horas, o que corresponde à realização de higiene oral pelo cirurgião-dentista e manutenção pela equipe de Enfermagem. A inserção de protocolos preventivos da pneumonia nosocomial, o monitoramento e a descontaminação da cavidade oral dos pacientes em UTI por profissionais qualificados, são a chave para a redução da colonização pulmonar por patógenos orais e, consequentemente, redução da incidência de pneumonias nosocomiais.
\end{abstract}

Palavras-chave: Odontólogos; Biofilme; Pneumonia; Unidade de Terapia Intensiva.

\begin{abstract}
This paper describes the oral condition of patients admitted to the intensive care unit (ICU) of a public hospital in Manaus-Amazonas with a diagnosis of nosocomial pneumonia. A cross-sectional, quantitative study was carried out in hospitalized patients aged over 18 years, unable to self-care and with a clinical diagnosis of pneumonia. The clinical evaluation of the oral cavity was divided into two moments: initial (up to 48 hours after admission) and after 72 hours. Plaque index (PI), mucosal condition, presence or not of dental prosthesis, number of teeth present and tongue coating index were evaluated. Of the twenty patients evaluated, $60 \%$ were female and the predominant age group was between 70 and 90 years old (35\%). 13 patients were on IMV (Induced Mechanical Ventilation) and 7 were on room air. There was an improvement in Plaque indexes (detection of dental biofilm) and presence of tongue coating after 72 hours, which corresponds to the performance of oral hygiene by the dentist and maintenance by the nursing team. The insertion of preventive protocols for nosocomial pneumonia, monitoring and decontamination of the oral cavity of patients in the ICU by qualified professionals are the key to reducing lung colonization by oral pathogens and, consequently, reducing the incidence of nosocomial pneumonia.
\end{abstract}

Keywords: Dentists; Biofilm; Pneumonia; Intensive Care Unit. 


\begin{abstract}
Resumen
Este artículo describe la condición bucal de los pacientes ingresados en la unidad de cuidados intensivos (UCI) de un hospital público de Manaus-Amazonas con diagnóstico de neumonía nosocomial. Se realizó un estudio transversal, cuantitativo, en pacientes hospitalizados mayores de 18 años, incapaces de autocuidado y con diagnóstico clínico de neumonía. La evaluación clínica de la cavidad bucal se dividió en dos momentos: inicial (hasta 48 horas después del ingreso) y después de 72 horas. Se evaluó el índice de placa (IP), el estado de las mucosas, la presencia o no de prótesis dental, el número de dientes presentes y el índice de recubrimiento de la lengua. De los veinte pacientes evaluados, el $60 \%$ eran mujeres y el grupo de edad predominante estaba entre 70 y 90 años (35\%). 13 pacientes estaban en IMV (ventilación mecánica inducida) y 7 estaban en aire ambiente. Hubo una mejoría en los índices de placa (detección de biofilme dental) y presencia de saburra en la lengua a las 72 horas, lo que corresponde a la realización de higiene bucal por parte del odontólogo y mantenimiento por parte del equipo de enfermería. La inserción de protocolos preventivos de neumonía nosocomial, el seguimiento y descontaminación de la cavidad bucal de los pacientes en UCI por profesionales cualificados son la clave para reducir la colonización pulmonar por patógenos orales y, en consecuencia, disminuir la incidencia de neumonía nosocomial.
\end{abstract}

Palabras clave: Odontólogos; Biopelícula; Neumonía; Unidad de Cuidados Intensivos.

\title{
1. Introdução
}

A dificuldade de autocuidado do paciente internado em UTI, bem como a redução de fluxo salivar são fatores predisponentes a instalação de patologias bucais. Estes fatores associados a condições pré-existentes como: Biofilme, cálculo dental, doença periodontal, lesões cariosas, necrose pulpar e traumas provocados por próteses; podem influenciar de maneira negativa a condição sistêmica do paciente e agravar seu quadro clínico (Rabelo et al., 2010; Gehring et al., 2021).

Uma das complicações mais comuns em pacientes críticos é a pneumonia nosocomial (PN). É considerada uma das condições responsáveis por altos índices de morbidade e mortalidade nos pacientes internados nas unidades de terapia intensiva. É uma das infecções hospitalares mais incidentes, repercutindo significativamente nos custos hospitalares e demora na alta do paciente (Beraldo \& Andrade, 2008; Simões et al., 2020).

A forma mais comum dos microrganismos bucais alcançarem o trato-respiratório é através da aspiração do conteúdo da orofaringe, inalação de aerossóis infectantes, disseminação de infecção de áreas adjacentes e contaminação hematogênica. Em pacientes com depressão do nível de consciência, o risco de pneumonia nosocomial se torna muito maior, pois muitas vezes estes pacientes ficam com a boca entreaberta ou mesmo aberta, além de estar propensos a aspirar conteúdo da orofaringe durante o sono (Amaral et al., 2009; Buisch et al., 2009).

A presença do biofilme dental pode desencadear uma resposta inflamatória, ocasionando um aumento significativo na quantidade de imunoglobulinas e de mediadores químicos de inflamação circulantes, trazendo prejuízos, tanto no local, quanto em sítios distantes, e dando suporte a uma relação entre a doença periodontal e doenças sistêmicas (Morais et al., 2006; Silva et al., 2017).

O cirurgião-dentista inserido na equipe multiprofissional visa oferecer ao paciente internado a assistência e tratamento odontológico, evitando que ocorram agravos do quadro clínico inicial e prevenção da propagação de infecções para outros órgãos e sistemas (Meira et al., 2010; Nawata et al., 2019).

Um dos pontos do pacote de cuidados (bundles) de prevenção à pneumonia nosocomial (Silva et al., 2012), está a higiene oral com Clorexidina $0,12 \%$. Neste contexto, é de suma importância a presença do cirurgião-dentista em ambiente hospitalar, em conjunto com os demais profissionais da equipe multidisciplinar. Este trabalho avaliou a condição clínica odontológica de pacientes com diagnóstico de Pneumonia, internados na UTI de um hospital público de Manaus/AM, considerando variados fatores como: a condição da mucosa, índice de saburra e presença de biofilme dental. O objetivo foi apresentar a correlação entre a condição bucal e a presença de Pneumonia e formas de prevenção pela equipe odontológica. 


\section{Metodologia}

Trata-se de estudo observacional, de cunho transversal, onde houve avaliação de uma população definida (pacientes internados em uma UTI pública) e em um tempo determinado (os dados foram coletados no período de setembro a novembro de 2015) (Estrela, 2018). Este trabalho foi aprovado pelo CEP, parecer CAAE: 48598015.5.0000.5512

Os responsáveis pelos pacientes internados, foram orientados sobre o objetivo da pesquisa e após a leitura do TCLE (Termo de Consentimento Livre e Esclarecido), autorizaram a coleta dos dados.

Participaram do estudo 20 pacientes, que atendiam aos critérios de inclusão a saber: pacientes internados em UTI, com idade superior a 18 anos, diagnóstico de pneumonia nosocomial e impossibilitado do autocuidado. A avaliação clínica odontológica foi realizada por apenas um pesquisador e considerando o índice de placa/presença de biofilme dental (IP) proposto por de Silness \& Löe (1964). Foi avaliado: a condição da mucosa, a presença ou não de prótese dentária, o número de dentes presentes e o índice de saburra lingual. A coleta dos dados foi realizada em dois momentos distintos: inicialmente em até 48 horas da internação, seguida por uma segunda avaliação após 72 horas da primeira, conforme protocolo já descrito na literatura (Cruz et al., 2014).

\section{Resultados}

O presente estudo avaliou 20 pacientes admitidos na UTI de um hospital público de Manaus que preencheram os critérios de inclusão da pesquisa. A maioria (60\%) dos pacientes pertencia do gênero feminino. 35\% dos pacientes possuíam entre 70 e 90 anos de idade. Quanto ao motivo da internação, 9 (45\%) dos 20 pacientes estavam internados por causa de complicações de doenças circulatórias (Tabela 1).

Tabela 1 - Dados Sociodemográficos.

\begin{tabular}{lll}
\hline Dados Gerais & n & \% \\
\hline Gênero & & \\
Feminino & 12 & 60 \\
Masculino & 8 & 40 \\
Faixa etária & & \\
18 a 30 & 5 & 25 \\
30 a 50 & 2 & 10 \\
50 a 70 & 6 & 30 \\
70 a 90 & 7 & 35 \\
Motivo da Internação & & \\
Doenças do aparelho respiratório & 7 & 35 \\
Doenças Endócrinas & 1 & 5 \\
Acidentes & 1 & 5 \\
Doença renal crônica & 2 & 10 \\
Doenças circulatórias & 9 & 45 \\
\hline
\end{tabular}

Fonte: Autores, Manaus (2021).

O Gráfico 1 apresenta o número de pacientes sob VM (Ventilação mecânica) e em ar ambiente. Dos vinte pacientes avaliados, 13 (65\%) encontravam-se intubados (VMI- Ventilação Mecânica Induzida), tendo sido observadas alterações bucais em 3 destes pacientes a saber: trauma, ressecamento e lesão em região de língua. A presença de xerostomia foi observada em todos estes pacientes. 
Gráfico 1 - Pacientes em uso de Ventilação Mecânica.

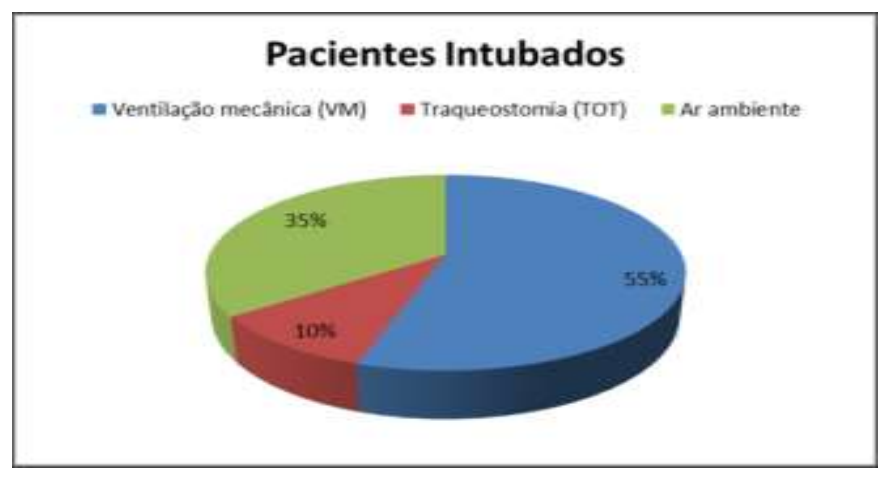

Fonte: Autores, Manaus (2021).

A Tabela 2 apresenta a detecção de biofilme dental, tendo sido avaliado através do Índice de Placa (IP). Na primeira avaliação, foi constatado que todos os pacientes apresentavam biofilme bucal, sendo que 10 pacientes (50\%) apresentavam IP de intensidade 2. Após 72 horas da primeira avaliação, houve melhora significativa e dos 10 avaliados na 1a inspeção, agora somente 5 pacientes $(25 \%)$ apresentavam biofilme visível.

Tabela 2 - Avaliação da presença de Biofilme Dental.

\begin{tabular}{lcc}
\hline \multicolumn{3}{c}{ Avaliação do Índice de Placa (IP)/ Presença de Biofilme dental } \\
\hline \multicolumn{1}{c}{ Intensidade } & $\begin{array}{c}\text { Primeira Avaliação } \\
\text { n (\%) }\end{array}$ & $\begin{array}{c}\text { Avaliação Após 72 Horas } \\
\text { n (\%) }\end{array}$ \\
\hline Intensidade 1* & $3(15 \%)$ & $8(40 \%)$ \\
Intensidade 2** & $10(50 \%)$ & $5(25 \%)$ \\
Intensidade 3*** & $7(35 \%)$ & $7(35 \%)$ \\
Total & $20(100 \%)$ & $20(100 \%)$ \\
\hline
\end{tabular}

Intensidade $1^{*}$ - não há placa por meio da simples visão. Há placa quando se realiza a passagem de sonda na área dento-gengival;

Intensidade $2 * *$ - há placas por meio da simples visão;

Intensidade $3^{* * *}$ - há placa bacteriana por meio da simples visão rodeando o dente,

inclusive nos espaços interdentais, podendo haver cálculos. (Cruz et al., 2014.

Fonte: Autores, Manaus (2021).

Quanto à avaliação da presença de Saburra Lingual, observamos que do total de pacientes avaliados, 8 pacientes (40\%) não apresentavam saburra lingual. Após 72 horas da primeira avaliação, nota-se na Tabela 3, que o índice de saburra lingual nos pacientes diminuiu em todos os pacientes avaliados.

Tabela 3 - Índice de Saburra Lingual.

\begin{tabular}{lcc}
\hline & Avaliação do Índice de Saburra Lingual \\
\hline Saburra lingual & $\begin{array}{c}\text { Primeira Avaliação } \\
\text { n (\%) }\end{array}$ & $\begin{array}{c}\text { Avaliação Após 72 Horas } \\
\text { n (\%) }\end{array}$ \\
\hline Saburra subclínica & $6(30 \%)$ & $4(20 \%)$ \\
Saburra $\leq \mathbf{1 / 3}$ & $5(25 \%)$ & $4(20 \%)$ \\
Saburra $\leq \mathbf{2} / \mathbf{3}$ & $1(5 \%)$ & $2(10 \%)$ \\
Sem Saburra & $8(40 \%)$ & $10(50 \%)$ \\
Total & $20(100 \%)$ & $20(100 \%)$ \\
\hline
\end{tabular}

Fonte: Autores, Manaus (2021).

A Tabela 4 apresenta a espessura da Saburra lingual. Na primeira avaliação, 10 pacientes (50\%) apresentaram saburra lingual fina, com papilas linguais visíveis; embora 40\% não possuíam saburra lingual espessa. Na segunda avaliação após as 72 
horas, observou-se em 8 pacientes (40\%) papilas linguais visíveis e 10 pacientes (50\%) não apresentaram saburra lingual espessa, o que demonstra melhora no quadro clínico.

Tabela 4 - Espessura da Saburra Lingual.

\begin{tabular}{lcc}
\hline \multicolumn{3}{c}{ Avaliação da Espessura da Saburra Lingual } \\
\hline Saburra lingual & $\begin{array}{c}\text { Primeira Avaliação } \\
\text { n (\%) }\end{array}$ & $\begin{array}{c}\text { Avaliação Após 72 horas } \\
\text { n (\%) }\end{array}$ \\
\hline Saburra fina, papilas linguais visíveis & $10(50 \%)$ & $8(40 \%)$ \\
Saburra espessa, papilas linguais sem visibilidade & $2(10 \%)$ & $2(10 \%)$ \\
Não apresentaram espessura & $8(40 \%)$ & $10(50 \%)$ \\
Total & $20(100 \%)$ & $20(100 \%)$ \\
\hline
\end{tabular}

Fonte: Autores, Manaus (2021).

Quanto ao número de dentes presentes na cavidade bucal desses pacientes, 6 pacientes (30\%) eram desdentados totais, e apenas 4 usavam prótese total. 8 pacientes $(40 \%)$ eram desdentados parciais e somente 1 destes, usava prótese parcial removível. 6 pacientes (30\%) eram dentados (Tabela 5).

Tabela 5 - Total de Elementos dentais Presentes.

\begin{tabular}{lc}
\hline \multicolumn{2}{c}{ Elementos dentais presentes e ausentes } \\
\hline & $\mathbf{n}(\%)$ \\
Dentado & $6(30)$ \\
Desdentado Total & $6(30)$ \\
Desdentado Parcial & $8(40)$ \\
Total & $20(100 \%)$ \\
\hline
\end{tabular}

Fonte: Autores, Manaus (2021).

\section{Discussão}

A Pneumonia é definida como uma infecção do trato respiratório inferior, sendo comum o seu diagnostico após quarenta e oito horas de internação do paciente não estando presente antes do momento da internação (Rezende et al., 2020). É frequente em pacientes hospitalizados sendo denominada pneumonia nosocomial (PN) quando tem início 48-72 horas ou mais após a admissão hospitalar. Classifica-se em precoce quando se inicia antes do quinto dia da admissão ou tardia quando ocorre após este período. Considerada como causa importante de mortalidade entre os pacientes internados, com uma taxa estimada de $30 \%$ (Pinheiro et al.,2007)

A pneumonia associada à ventilação mecânica (PAVM) é a infecção nosocomial mais comum na UTI. Tem prevalência variável, apresentando taxas desde 6 até 50 casos por 100 admissões na unidade de terapia intensiva (UTI). O desenvolvimento de pneumonia nosocomial, especificamente da PAVM, tem morbidade significativa associada, prolonga o tempo de ventilação mecânica, bem como o tempo de permanência na UTI, com todos os custos associados a esse prolongamento (Dalmora et al., 2013)

Os Pacientes em VM apresentam diminuição da produção salivar e impossibilidade de mastigação. Estes fatores, associados ao reflexo de tosse diminuído, pouca capacidade de higienização e deficiências físicas favorecem o acúmulo de biofilme dental, que pode atuar como reservatório para patógenos e que, se bronco aspirados, podem causar a Pneumonia nosocomial (Simões et al., 2020). Somado a isto, está o fato de os pacientes estarem imunologicamente comprometidos, devido a doenças ou medicamentos, e sujeitos à colonização por microrganismos multirresistentes (Amaral et al., 2009).

O Gluconato de Clorexidina 0,12\% têm sido preconizado como um antisséptico de escolha na realização da Higiene Oral de paciente em VM, representando uma alternativa para o controle químico e formação do biofilme dental. É um agente 
antimicrobiano de amplo espectro, capaz de atuar contra microrganismos gram-positivos e gram-negativos, incluindo os resistentes. Apresenta também, ação contra alguns fungos e vírus. Possui outra propriedade importante: a substantividade. A ação consiste em o antisséptico após sua aplicação na cavidade oral, aderir as superfícies dentais e mucosa, e ser lentamente liberado em até 12 horas após sua aplicação (Félix, 2016; Berg, 2019; Rezende et al., 2020).

Em nosso estudo, o acúmulo de biofilme teve melhora significativa na $2^{\mathrm{a}}$ avaliação, o que pode ser justificado pela presença de cirurgião-dentista capacitado para esta finalidade, na equipe multiprofissional da UTI objeto do estudo. A higiene oral é realizada diariamente com o uso de Clorexidina $0,12 \%$ como antisséptico bucal e escova dental. A higiene oral entra na prescrição do paciente e é realizada pela equipe de Enfermagem, devidamente treinada para isto. O uso da clorexidina $0,12 \%$ diminuíram a incidência de infecções respiratórias, a necessidade de antibióticos sistêmicos e a mortalidade de pacientes em ambiente hospitalar e UTI (Van Strydonck et al., 2005; Santos et al., 2013).

A saburra lingual é uma alteração comum em ambientes internados em UTI. É constituída por restos alimentares, células descamadas, fungos, bactérias e enzimas ativas que participam do processo da digestão (Miranda et al., 2018). Notamos que o depósito de saburra lingual já ocorre em poucas horas após a internação. Nota-se também que na $2^{\mathrm{a}}$ avalição, houve diminuição do número de pacientes com saburra visível. Isto se deve a limpeza mecânica da superfície lingual com escova dental ou limpadores de língua associado com Clorexidina $0,12 \%$ (limpeza química). Este procedimento desorganiza e remove a saburra lingual tanto em pacientes sob VM, quanto em pacientes em ar ambiente e deve fazer parte da rotina de higiene oral entre os pacientes 'Gomes \& Esteves, 2012; Miranda et al., 2018).

Em relação à condição dental dos pacientes avaliados, 6 pacientes (30\%) eram desdentados totais, e apenas 4 usavam prótese total. 8 pacientes (40\%) eram desdentados parciais e somente 1 destes, usava prótese parcial removível. 6 pacientes (30\%) eram dentados. Pacientes com dentes naturais possuem chance de ter um maior acúmulo de biofilme, inclusive nas áreas Inter proximais, o que acaba por levar ao desenvolvimento de pneumonia com maior frequência, se comparados com os desdentados totais (Cruz et al., 2014).

No nosso trabalho, 4 dos 6 pacientes foram internados e intubados com próteses Total. Se não higienizadas adequadamente, as próteses funcionam como um reservatório e acúmulo de biofilme e microrganismos. Nos usuários de prótese, a Cândida é o microrganismo e fungo mais presente, o que pode levar consequentemente ao desenvolvimento de doenças sistêmicas e pneumonia nosocomial. A equipe de Enfermagem deve ser orientada a comunicar ao cirurgião-dentista sobre a presença de próteses totais, além de receber orientações sobre a higienização e acondicionamento das mesmas (Fonseca et al., 2019).

\section{Considerações Finais}

A presença do cirurgião-dentista em equipe multiprofissional atuante em ambiente hospitalar, bem como a adoção de protocolos de higiene oral na rotina de atendimento ao paciente internado, atua de maneira conjunta e efetiva na redução do biofilme dental.

\section{Referências}

Amaral, S. M., Côrtes, A. Q., \& Pires, F. R. (2009). Nosocomial pneumonia: importance of the oral environment. Jornal Brasileiro de Pneumologia, 35(11):111624.

Beraldo, C. C., \& Andrade, D. (2008). Higiene bucal com clorexidina na prevenção de pneumonia associada à ventilação mecânica. Journal Brasileiro de Pneumologia. 34 (9): 707-714.

Berg, T. L. (2019). Impacto da atuação do cirurgião-dentista em um Bundle de pneumonia associada à ventilação mecânica de uma Unidade de Terapia Intensiva 52 f.: il. Dissertação (Mestrado Profissional Pesquisa em Saúde) - Centro Universitário CESMAC, Programa de Pós-Graduação em Pesquisa em Saúde, Maceió - AL, 2019. 
Buischi, Y. P., Axelsson, P., Siqueira, \& T. R. F. Controle mecânico do biofilme dental e a prática da promoção de saúde bucal. In: Buischi, Y.P. (2009). Promoção de saúde bucal na clínica odontológica. Artes Médicas.

Cruz, M. K., Morais, T. M. N., Trevisani, D. M. (2014). Avaliação clínica da cavidade bucal de pacientes internados em unidade de terapia intensiva de um hospital de emergência. Revista Brasileira de Terapia Intensiva, 26 (4): 379-383.

Dalmora, C. H., Deutschendorf, C., Nagel, F., Santos, R. P., \& Lisboa, T. (2013). Definindo pneumonia associada à ventilação mecânica: um conceito em (des)construção, Revista Brasileira de Terapia Intensiva, 25(2):81-86

Estrela, C. Metodologia científica: ciência, ensino, pesquisa. (3a ed.), Artes Médicas, 2018.

Félix, L. C. (2016). Avaliação do impacto de dois diferentes métodos de higiene oral com clorexidina na prevenção de pneumonia associada à ventilação mecânica. 59 f. Dissertação (Mestrado em Odontologia) - Faculdade de Farmácia, Odontologia e Enfermagem, Universidade Federal do Ceará, Fortaleza, 2016.

Fonseca, E. O. S., Pedreira, L. C., Gomes, N. P., Amaral, J. B., Virgens, I. R., \& Santos, F. C. (2019). O cuidado de enfermagem no acondicionamento da prótese dentária de idosos hospitalizados. Acta Paulista de Enfermagem, 32(4):442-8.

Gehring, G. M., Costa, M. A. C., Cardoso, R. B., Souza, A. C. M. F., \& Bortoluzzi, M. C. (2021). Saúde oral e incidência de infecção nosocomial e mortalidade em pacientes submetidos a cirurgia cardíaca eletiva. Research, Society and Development, 10(5): e14910514658.

Gomes, S. F., \& Esteves, M.C.L. (2012). Atuação do cirurgião-dentista na UTI: um novo paradigma. Revista Brasileira de Odontologia, 69(1): 67-70.

Meira, S. C. R., Oliveira, C. A. S., \& Ramos, I. J. M. (2010). A importância da participação do cirurgião-dentista na equipe multiprofissional hospitalar. $9^{a}$ edição do prêmio SINOG de Odontologia. Curso de Odontologia do Centro Universitário Newton Paiva, Belo Horizonte/MG.

Miranda, A. F., El Haje, G. L. C., de Paula, R. M., Costa, P. P., \& Biazevic, M. G. H. (2018). Assessing the incidence of tongue coating in patients treated in intensive care units. Revista Brasileira de Odontologia, 75(e1213):1-5.

Morais, T. M. N., Silva, A., Avi, A. L. R. O., Souza, P. H. R., Knobel, E., \& Camargo, L. F. A. (2006). A importância da atuação odontológica em pacientes internados em unidade de terapia intensiva. Revista Brasileira de Terapia Intensiva, 18(4): 412-417.

Nawata, W., Umezaki' Y., Yamaguchi, M., Nakajima, M., Makino, M., Yoneda' M., Hirofuji, T.,Takafumi Yamano, T., Ooboshi, H., \& Morita, H. (2019). Continuous Professional Oral Health Care Intervention Improves Severe Aspiration Pneumonia. Case Report Dentistry, 16:1-6.

Pinheiro, P. G., Salani, R., Aguiar, A. S. W., \& Pereira, S. L. S. (2007). Perfil Periodontal de Individuos Adultos Traqueostomizados com Pneumonia Nosocomial. Revista de Periodontia, 17 (3):67-72.

Rabelo, G. D., Queiroz, C. I., \& Santos, P. S. S. (2010). Atendimento odontológico ao paciente em unidade de terapia intensiva. Arquivos Medicos dos Hospitais e da Faculdade de Ciencias Médicas da Santa Casa São Paulo. 55(2): 67-70.

Rezende, R. P., Vieira, V. C., Falcão, G. G. V. S. C., Ribeiro, P. L., \& Sarmento, V. A. (2020). Uso da Clorexidina na Prevenção da Pneumonia Nosocomial em Pacientes Internados em UTI: Revisão Sistemática. Revista da Faculdade de Odontologia da Universidade Federal da Bahia, 50(1): 1-8.

Santos, P. P. S., Mariano, M., Kallas, M. S., \& Vilela, M. C. N. (2013). Impacto da remoção de biofilme lingual em pacientes sob ventilação mecânica. Revista Brasileira de Terapia Intensiva, 25 (1):44-48.

Silness, J., \& Löe, H. (1964). Periodontal Disease in Pregnancy. Ii. Correlation between Oral Hygiene and Periodontal Condtion. Acta Odontologica Scandinavica, (22): 121- 135 .

Silva, I. O., Amaral, F. R., da-Cruz, P. M., \& Sales, T. O. (2017). A importância do cirurgião-dentista em ambiente hospitalar. Revista Médica de Minas Gerais, $27(\mathrm{e}-1888): 1-5$

Silva, S. G., Nascimento, E. R. P., \& Salles, R. K. (2012). Bundle de prevenção da pneumonia associada à ventilação mecânica: uma construção coletiva. Texto contexto - Enfermagem, 21 (4): 837-844.

Simões, T. M. S., Fernandes Neto, J. de A., Ferreira, A. C. D., Sousa, J. A. de, Medeiros, C. L. S. G. de. \& Catão, M. H. C. de V. (2020). Controle do biofilme oral e sua relação com a redução de infecções respiratórias em pacientes de UTI: uma revisão de ensaios clínicos. Research, Society and Development, 9(10): e8339108594.

Van Strydonck, D. A., Timmerman, M. F., van der Velden, U., \& van der Weijden, G. A. (2005). Plaque inhibition of two commercially available chlorhexidine mouthrinses. Journal of Clinical Periodontology, 32(3):305-9. 